Nig J. Biotech. Vol. 31 (2016) 27 -39

ISSN: 01891731

Available online at

http://www.ajol.info/index.php/njb/index

and www.biotechsocietynigeria.org

DOI: http://dx.doi.org/10.4314/njb.v31i1.5

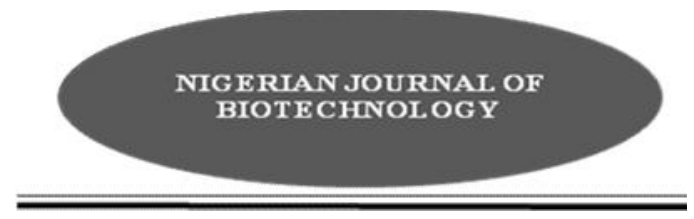

\title{
Comparative effects of pretreatment of stem cuttings of Chromolaena odorata (Siam weed) with sodium azide and hydroxylamide on the survival and phyoremediative performance in an oil-polluted soil
}

\author{
I khajiagbe, B. ${ }^{1}$ and Akindolor, A. ${ }^{2}$ \\ ${ }^{1}$ Department of Plant Biology and Biotechnology, Univ. of Benin, Benin City, ${ }^{2}$ Department of Environ. \\ Mgt and Toxicology, Univ. of Benin, Benin City.
}

(Received: 23:12:2015; Accepted: 14:07:2016)

\begin{abstract}
The method of phytoremediation was applied to clean up heavy metals and polyaromatic hydrocarbon contents of a waste engine oil (WEO)-polluted soil, using Chromolaena odorata exposed to sodium azide $\left(\mathrm{NaN}_{3}\right)$ and hydroxylamine hydrochloride $\left(\mathrm{NH}_{2} \mathrm{OH} . \mathrm{HCl}\right)$ solutions respectively. WEO was poured into sun-dried top soil, and thoroughly mixed to obtain $5 \% \mathrm{w} / \mathrm{w}$ concentration in soil. After one month, soil was sown with sodium azide and hydroxylamine hydrochloride pretreated stem cuttings of $\mathrm{C}$. Odorata. Concentrations of either of the mutagenic agents were $0.016 \%, 0.064 \%$, and $0.25 \%$ respectively. Results showed that there was significant decrease in heavy metal and polyaromatic hydrocarbon (PAH) components of soil. Remediation of $\mathrm{Cu}$ and $\mathrm{Pb}$ by $\mathrm{C}$. Odorata was best at $0.25 \%$ $\mathrm{NH}_{2} \mathrm{OH} . \mathrm{HCl}$ treatment. Remediation efficiencies for PAH and heavy metals were better enhanced with the pretreatment of Chromolaena odorata with mutagenic agents. Results also showed that with the increase in concentration of mutagenic solutions required for presoaking, total heavy metal concentration in soil decreased. PAH remediation efficiency was highest (89.88 - 90.99\%) when soils were remediated with sodium azide-treated plants than their hydroxylamine hydrochloride counterparts (72.54 - 81.14\%). Similar observations were made in heavy metal reduction rates.
\end{abstract}

Keywords: phytoremediation, sodium azide, hydroxylamine hydrochloride, heavy metals, aromatic hydrocarbon

Correspondence: ikhaj@yahoo.com

\section{Introduction}

The world is one global village with industrialization the building block or backbone of most nations' economy. In Nigeria, industrialization is powered by the petroleum industry, while creating economic boom it has led to environmental and socio-economic problems (Raven et al., 1993; Ibekwe et al., 2006). Spills from oil are devastating to plants and animals found in the soil or which inhabit soils, because of their toxicity and also because the presence of hydrocarbon reduce oxygen tension and increase anaerobiosis in the soil which is damaging to plant roots (Bossert and Bartha, 1984).

One of the major products of petroleum is engine oil or lubricating oil. Engine oil helps to reduce friction between moving parts of auto machines and engines, prevents corrosion of these auto machines parts. It contains additive chemical substance such as amines, benzene, phenols, barium, lead, zinc, sulphur, magnesium and phosphorus and polyaromatic hydrocarbons and synthetic poly-chlorinated biphenyls. According to Wang et al. (2002), engine oil got from automobile (covering up to $3,000 \mathrm{~km}$ ) contains significantly higher concentrations of polyaromatic hydrocarbon (PAH), compared to new lubricating oil which contains only low concentration of polyaromatic hydrocarbon. A major source of oil pollution in the environment is as a result of disposal or discharge of used engine oil from vehicles. Extintion or total death of soil ecosystem and its habitat of living organism is an effect of oil pollution on soil (Akoachere et al., 2008).

The common practice by most automobile operators and service men of disposal of used engine oil into watercourse, 
gutters, farmland and open vacant plots has increased the incidence of oil contamination to agricultural lands. Heavy metals such as vanadium, lead, nickel and iron, present in spent engine are of high value while that present in unused or new engine oil is relatively low (Whisman et al., 1974). These oil contaminated soils are serves as potential source for surface and ground water contamination, beside the fact that they are unsuitable for agricultural and recreational purposes.

Lands or soils exposed to oil pollution are damaged and made infertile. Crude oil, as well as its products, in soil results in poor soil fertility (Odu, 1972). oil polluted soil has been reported to have great impact on plant. Soil pollution is the introduction of contaminant into the soil matrix, which hinder or reduce the activites of organism present in the soil. The most common type of contaminants are chemicals which could be organic such as hydrocarbon, polyaromatic hydrocarbon (PAHs), polychlorinated biphenyls (PCBs), chlorinated compounds, detergents and pesticides. Inorganic such as nitrates, phosphates and heavy metals such as cadium, lead and vanadium.

Different remediation methods exist for the removal of these contaminants. However, most of these methods are not enviromental suitable as they release or add contaminant to the environment. Among these methods for remediation of oil pollution is phytoremediation i.e the use of plant to clean up or degrade contaminant from the environment. Phytoremediation is an effective, nonenvironmental contaminanting process and low cost as most plants used for used for phytoremedation is easily accessible with one or two food crop. All plants which have phytoremediative capabilities are usually tolerant to the contaminants which they remediate. A number of researchers conducted, stated that Chromolaena odorata has the ability to tolerate soil acidity, aluminum saturation and oil (Anoliefo and Vwiko, 2001). Exampls of plant species used for phytoremediation are Willow, Poplar, Soybean, Sunflower, Indian mustard, Red clover, Chromolaena odorata e.t.c. Chromolaena odorata (commonly called siam weed, Awolowo or shell plant in Nigeria) has been found to have phytoremediation properties, it degrade contaminant from soil when cultivated or found naturally growing as weeds on a polluted site. The use of plants in the reclamation of contaminated lands is called phytoremediation. This process maintains soil structure, is environmental friendly and is low cost (Khan et al., 2000). Pollutants which can be degraded by phytoremediation are ot two types, the elemental pollutants and the organic pollutants (Meagher, 2000).

Evidently, phytoremediative capabilities of most plants is a factor of its improved morphological presentations, it is suffices to say therefore, that any process that is adopted to enhance the growth, performance and morphological presdentations of the plants, would also enhance phytoremediation. One of such methods is the use of chemical mutagens (Mshembulla et al., 2012; Ikhajiagbe et al., 2013). The addition of certain chemical substance such as hydroxylamide and sodium azide, which have mutagenic properties has been known to increase plant growth and yield. There is significant increase in crop production due to mutation induction (Kharkwal and Shu, 2009) and the induction of desired attributes. This research, therfore aims to access the effect of these chemical reagents (hydroxylamide, sodium azide) on Chromolaena odorata as a phytoremediation agent. This outcome of this findings will be useful in the growing or cultivation of Chromolaena odorata on contaminated soils in Nigeria and other countries where Chromolaena species thrive.

\section{Materials and Method}

Top soil $(0-10 \mathrm{~cm})$ was collected, sun dried to constant weight and a measured $20 \mathrm{~kg}$ of the sun-dried soil was measured into experimental boxs. Waste engine oil was measured $(1 \mathrm{~kg} /$ bucket, specific gravity = 0.846 ) and poured into the soil, it was mixed thoroughly to obtain a $5 \% \mathrm{w} / \mathrm{w}$ oil- in-soil concentration.

$\mathrm{NaN}_{3}$ and $\mathrm{NH}_{2} \mathrm{OH} . \mathrm{HCl}$ solutions: Different concentrations of sodium azide and hydroxylamine solutions were prepared and used in the experiment. Percentage concentrations for sodium azide and hydroxylamide solutions included; $0.016 \%$, $0.064 \%$, and $0.25 \% \mathrm{w} / \mathrm{v}$, for both chemicals (designated in the study as PS1, PS2, and PS3 for sodium azide treatments and PX1, PX2, and PX3 for hydroxylamine treatments respectively).These respectives concentrations were obtained by weighing $0.16 \mathrm{~g}, 0.64 \mathrm{~g}$ and $2.5 \mathrm{~g}$ each of sodium azide $\left(\mathrm{NaN}_{3}\right)$ and hydroxylamine hydrochloride $\left(\mathrm{NH}_{2} \mathrm{OH} . \mathrm{HCL}\right)$, and dissolved in 1000 mlof distilled water $(\mathrm{pH}$ 7) respectively. 
Pretreatment of stem cuttings of Chromolaena odorata: Stem cuttings were collected from a fallow land located within the University of Benin Ugbowo Campus, Benin City, Nigeria. Thirty (30) centimeter-long stems, of relatively equal sizes were obtained. Twelve stem cuttings ( i.e 3 stem cuttings per treatment for 4 replicates) were placed in bowls containing each of the mutagenic solutions already prepared. Stems were completely submerged in the solutions for 1 hour with regular stirring every 15 minutes, to ensure equal exposure of stems.

Sowing: The research set up was made up of 8 treatments, each of which was replicated 4 times, making total of 32 experimental units. The experimental boxs were labeled and arranged according to treatment used for identification ease. After exposure, pretreated stems were immediately sown in the designated treatment experimental boxs, having priorly exposed the soil to 1 month of oil polution. This was to allow for natural attenuation of the soil before sowing. The set up was left for additional 2 months, after which soil was analysed for heavy metal and PAH contents of soil, whereas some plant growth parameters were also observed.

Extraction of Micronutrients in Soils by Hydrochloric Acid Method: Ten (10) g of soil was weighed into a $250 \mathrm{ml}$ plastic bottle. 100 $\mathrm{ml}$ of $0.1 \mathrm{~m} \mathrm{HCl}$ was added, stoppered, and then shaken for 30 minutes. The mixture was filtered through Whitman filter paper No.42. Following this - iron, copper, manganese, cadmium, chromium, lead, nickel and vanadium were determined in the filtrate by Atomic Absorption Spectrometery, Model Solaar 969 Unicam Series. An air-acetylene flame was used.

Determination of Polyaromatic Hydrocarbon Contents of Polluted Soil by Gas Chromatography (GC): A $10 \mathrm{~g}$ sample was extracted with methylene chloride (DCM). The extract was filtered through anhydrous sodium sulphate to remove any trapped water molecule. This was followed by a clean- up/ fractionation of the sample extract into aromatic (PAH) components. Finally, the components were concentrated using a rotary evaporator for GC analysis, using FID as detector. GC results used was calculated as follows:

Sample $(\mathrm{mg} / \mathrm{kg})=\quad$ Area $\times$ F.vol $\times 1000$ Rf $x$ Wt
$\mathrm{Rf}=$ Response factor $=$ Total Area $/$ Total Concentration, obtained from instrument calibration with standards.

Area is obtained from the chromatogram output.

F.vol is the final volume of the concentrated extract (in $\mathrm{ml}$ )

Wt is the initial weight of the homogenized sample (in grams) Identification of Soil Microorganisms: The soil samples were air-dried and sieved through a $2 \mathrm{~mm}$ mesh to remove undwanted material. One gram $(1 \mathrm{~g})$ of the soil was transfered to nine $(9 \mathrm{ml})$ millimetres of sterile distilled water in sterile glass containers to serve as serial dilution. The glass containers were shaken for 5 minutes and was taken as $10-1$ dilution factor, $10 \mathrm{ml}$ were then transferred from the 10-1 dilution into another $9 \mathrm{ml}$ blank to obtain a 10-2 dilution and same process of transfer was repeated twice to obtain a dilution factor of 10-4. The spread plate method was employed in taking the heterotrophic bacteria counts. One (1) $\mathrm{ml}$ of the serially diluted portion of $10-4$ of each soil sample was inoculated onto nutrient agar plates for bacteria and Potato dextrose agar plates for fungal counts. The plates were inoculated at room temperature for 24 hours and 72 hours respectively, for bacteria and fungi growth. After incubation colonies were then counted and the colony forming unit (cfu/g) of the soil samples determined.

Isolation of Bacterial and Fungal Oil Degraders: Bushnell- Haas (BH) medium $\left(\mathrm{MgSO}_{4}, 0.20 \mathrm{~g} / \mathrm{l} ; \mathrm{CaCl}_{2}, 0.02 \mathrm{~g} / \mathrm{l} ; \mathrm{K}_{2} \mathrm{H}, \mathrm{PO}_{4}, 1\right.$ $\mathrm{g} / \mathrm{l} ; \mathrm{NH}_{4} \mathrm{NO}_{3}, 1 \mathrm{~g} / \mathrm{l} ; \mathrm{FeCl}_{3}, 0.05 \mathrm{~g} / \mathrm{l} ; \mathrm{KH}_{2} \mathrm{PO}_{4}, 1$ $\mathrm{g} / \mathrm{l} ; \mathrm{pH}$ 7.0, was used as the enrichment medium with $8 \%(\mathrm{v} / \mathrm{v})$ filter sterilized oil as the sole carbon source. The medium was dispensed into in $100 \mathrm{ml}$ Erlenmeyer flasks and autoclaved at $121{ }^{\circ} \mathrm{C}$ for 15 minutes. Thereafter, $5 \mathrm{~g}$ of each soil sample was inoculated into each flask of the medium and incubated at $130 \mathrm{rpm}$ at room temperature in a HY-4 multifunctional shaker (B. Bran Scientific and Instrument Company, England). After 10 days, $1 \mathrm{ml}$ of enriched media was transferred into freshly prepared enrichment media and incubated under the same conditions as described above. Serial dilutions from the third enrichment process were inoculated onto nutrient agar plates and potato dextrose agar plates for oil-degrading bacterial and fungal counts respectively using the methods described by Cowan and Steel (1974) and Cheesebrough (1998).

Where, 
Determination of Growth and Yield Parameters: Six measurable growth parameters were obsereved in the study, including shoot height, number of sprouted branches, total number of leaves per plant, average length of sprouted branch, as well as leaf area.

Statistics: Analysis of variance in completely randomized design was done using the SPSS-15 statistical software, and means were separated by using the Least Significant Difference. Other statistical determinations for ecotoxicological assessments included Hazard Quotient (HQ) and Toxic Equivalency (TEQ) for PAH.

$\mathrm{HQ}=$ Measured concentration

Toxicity reference value or selected screening benchmark.

When $\mathrm{HQ}>1$ : Harmful effects are likely due to contaminant in question

When $\mathrm{HQ}=1$ : Contaminant alone is not likely to cause ecological risk

When $\mathrm{HQ}<1$ : Harmful effects are not likely

$\mathrm{TEQ}=\Sigma \mathrm{Ti} \times \mathrm{PEF}$

Where $T E Q=$ Toxic Equivalency $\mathrm{Ti}=$ PAH concentration in soil $\mathrm{PEF}=$ Potency Equivalency factor

\section{Results}

The chemical composition of materials used for the experiment is indicated in Table 1. Heavy metal concentration of $\mathrm{Mn}, \mathrm{Ni}$ and $\mathrm{V}$ were below detection levels in all the treatments (Table 2). Similarly, soils with sodium azide-treated plants were totally remediated of $\mathrm{Cr}$, compared to a range of 7.29 - $16.51 \mathrm{mg} / \mathrm{kg}$ in other treatments. Results also showed that with the increase in concentration of mutagenic solutions required for presoaking, total heavy metal concentration in soil decreased. Total heavy metal was least in PS3 $(16.17 \mathrm{mg} / \mathrm{kg})$, compared to 21.04 $\mathrm{mg} / \mathrm{kg}$ as total in PS1, $28.43 \mathrm{mg} / \mathrm{kg}$ in PX1 and $20.43 \mathrm{mg} / \mathrm{kg}$ in PX3.

PAH components of soil exposed to experimental treatments is presented on Table 3. Total PAH at 1 month after pollution and just before plants were sown was $916.91 \mathrm{mg} / \mathrm{kg}$. PAH remediation efficiency of the sown $\mathrm{NaN}_{3}$-treated stem cuttings (i.e. PS) in the oil-polluted soils was highest (89.38 $90.99 \%)$, compared to those remediation efficiency of $\mathrm{NH}_{2} \mathrm{OH}$. $\mathrm{HCl}$-treated stem cuttings (PX-soils) (72.54 to 81.14\%). Naphthalene, acenaphthylene, 2-bromonaphthalene, acenaphthene, benzo(a)anthracene and chrysene were all remediated below detection $(<0.0001 \mathrm{mg} / \mathrm{kg})$ in all PS-soils. However PXsoils treatment contained detectable concentrations of the respective $\mathrm{PAH}$ components $(0.03-9.69 \mathrm{mg} / \mathrm{kg})$. There was also total remediation of naphthalene, acenaphthylene, 2-bromonaphthalene, acenaphthene, benzo(a)anthracene and chrysene in both PX1 and PX2. Comparatively $\mathrm{PAH}$ remediation were better enhanced in the soil sown with PS-treated plants.

Hazard quotients (HQ) to accertain ecological toxicity of residual concentration of heavy metal and PAH fractions are presented on Table 4. HQ was less than1 through out the duration of the experiment for $\mathrm{Cu}, \mathrm{Mn}, \mathrm{Ni}, \mathrm{V}$ and $\mathrm{Pb}$, an indication that ecological toxicity may not be indicated as a result of the presence of these heavy metals. However, HQ for $\mathrm{Cr}$ was greater than1 just before chromoleana odorata was sown (18.33) and at 2 MAP after untreated plant was sown. HQ was zero in PS-soils, this signified a significant remediation of $\mathrm{Cr}$ from toxic level to ecologically harmless level. Pretreatement of $\mathrm{NH}_{2} \mathrm{OH} . \mathrm{HCl}$ plants did not enhance phytoremediation of $\mathrm{Cr}$ after two months, compared to pretreatment with $\mathrm{NaN}_{3}$. $\mathrm{HQ}$ to accertain toxicity of heavy metals to soil microorganism and microbial process was less than 1 for $\mathrm{Cu}, \mathrm{Mn}, \mathrm{Ni}, \mathrm{V}$ and $\mathrm{Pb}$ through out the experiment (Table 4). When HQ is greater than 1, ecotoxic effects were due to that particular contaminant. HQ was greater than 1 in the PAH components evaluated for PX3 soil as well as in the soil that had the untreated plants.

The TEQ concentration of Benzo(a)anthracene was $5.38 \mathrm{mg} / \mathrm{kg}$ at Day 1 , and 5.52 at 1 MAP. However, this reduced to zero in PS-soil as well as PX1 and PX2 after 2 months (Table 5). Total TEQ at Day 1 was $238.72 \mathrm{mg} / \mathrm{kg}$ compared to $243.79 \mathrm{mg} / \mathrm{kg}$ in the soil sown with untreated plant at $3 \mathrm{MAP} / 2$ MAS. However, TEQ in PS-soil ranged from 19.58 to $21.33 \mathrm{mg} / \mathrm{kg}$ and from 32.74 to $61.74 \mathrm{mg} / \mathrm{kg}$ in PX-soil. When TTEQ for concentration of $\mathrm{PAH}$ mixture exceeds the method B clean up levels, the clean up level for Benzo(a)pyrene(0.137) was no meant for the level. 
Table 1: Chemical composition of materials used for the experiment.

\begin{tabular}{lll}
\hline Parameters & Soil $(\mathrm{mg} / \mathrm{kg})$ & Waste oil $(\mathrm{mg} / \mathrm{kg})$ \\
\hline Naphthalene & $\mathrm{Bd}$ & 26.58 \\
Acenaphthylene & $\mathrm{Bd}$ & 7.98 \\
2-bromonaphthalene & $\mathrm{Bd}$ & 29.54 \\
Acenaphthene & $\mathrm{Bd}$ & 26.32 \\
Fluorine & $\mathrm{Bd}$ & 42.04 \\
Phenanthrene & 0.85 & 3.68 \\
Anthracene & $\mathrm{Bd}$ & 21.57 \\
Fluoranthene & $\mathrm{Bd}$ & 32.68 \\
Pyrene & $\mathrm{Bd}$ & 23.98 \\
Benzo(a)anthracene & $\mathrm{Bd}$ & 42.05 \\
Chrysene & $\mathrm{Bd}$ & 106.54 \\
Benzo(b,j,k)fluoranthene & $\mathrm{Bd}$ & 41.68 \\
Benzo(a)pyrene & 40.28 & 129.87 \\
Indeno(1,2,3-cd)pyrene & 5.24 & 129.54 \\
Dibenzo(a,h)anthracene & 12.25 & 34.68 \\
Benzo(g,h,i)perylene & 19.24 & 63.25 \\
& & \\
Copper, Cu & $\mathrm{Bd}$ & 7.92 \\
Manganese, Mn & $\mathrm{Bd}$ & $\mathrm{Bd}$ \\
Nickel, Ni & $\mathrm{Bd}$ & $\mathrm{Bd}$ \\
Vanadium, V & $\mathrm{Bd}$ & $\mathrm{Bd}$ \\
Chromium, Cr & 0.08 & 16.85 \\
Lead, Pb & $\mathrm{Bd}$ & 9.96 \\
\hline & &
\end{tabular}

$\mathrm{Bd}$ - below detectable limit of $0.0001 \mathrm{mg} / \mathrm{kg}$.

Table 2: Heavy metal composition of soil subjected to various treatments

\begin{tabular}{|c|c|c|c|c|c|c|c|c|c|c|c|}
\hline \multirow{3}{*}{$\begin{array}{l}\text { Heavy } \\
\text { metals } \\
(\mathrm{mg} / \mathrm{kg})\end{array}$} & \multirow{2}{*}{$\begin{array}{c}\text { Polluted } \\
\text { soil at } \\
\text { Day } 1\end{array}$} & \multirow{2}{*}{$\begin{array}{c}1 \text { month } \\
\text { after } \\
\text { pollution }\end{array}$} & \multicolumn{8}{|c|}{3 months after pollution } & \multirow{3}{*}{$\begin{array}{c}\text { LSD } \\
(0.05)\end{array}$} \\
\hline & & & Unpolluted & $\begin{array}{c}\text { Polluted } \\
\text { soil }\end{array}$ & PS1 & PS2 & PS3 & PX1 & PX2 & PX3 & \\
\hline & \multicolumn{10}{|c|}{$(\mathrm{mg} / \mathrm{kg})$} & \\
\hline $\mathrm{Cu}$ & 16.23 & 14.03 & $\mathrm{Bd}$ & 12.21 & 7.18 & 6.02 & 5.19 & 7.03 & 6.38 & 5.02 & 1.87 \\
\hline $\mathrm{Mn}$ & $\mathrm{Bd}$ & $\mathrm{Bd}$ & $\mathrm{Bd}$ & $\mathrm{Bd}$ & $\mathrm{Bd}$ & $\mathrm{Bd}$ & $\mathrm{Bd}$ & $\mathrm{Bd}$ & $\mathrm{Bd}$ & $\mathrm{Bd}$ & NA \\
\hline $\mathrm{Ni}$ & $\mathrm{Bd}$ & $\mathrm{Bd}$ & $\mathrm{Bd}$ & $\mathrm{Bd}$ & $\mathrm{Bd}$ & $\mathrm{Bd}$ & $\mathrm{Bd}$ & $\mathrm{Bd}$ & $\mathrm{Bd}$ & $\mathrm{Bd}$ & NA \\
\hline V & $\mathrm{Bd}$ & $\mathrm{Bd}$ & $\mathrm{Bd}$ & $\mathrm{Bd}$ & $\mathrm{Bd}$ & $\mathrm{Bd}$ & $\mathrm{Bd}$ & $\mathrm{Bd}$ & $\mathrm{Bd}$ & $\mathrm{Bd}$ & NA \\
\hline $\mathrm{Cr}$ & 20.36 & 18.33 & 0.02 & 16.51 & $\mathrm{Bd}$ & $\mathrm{Bd}$ & $\mathrm{Bd}$ & 10.56 & 9.21 & 7.29 & 2.63 \\
\hline $\mathrm{Pb}$ & 18.39 & 16.85 & $\mathrm{Bd}$ & 13.68 & 13.86 & 13.05 & 10.98 & 10.84 & 9.08 & 8.12 & 4.32 \\
\hline Total & 54.98 & 49.21 & 0.02 & 42.40 & 21.04 & 19.07 & 16.17 & 28.43 & 24.67 & 20.43 & - \\
\hline
\end{tabular}

$\mathrm{Bd}$ - below detectable limit of $0.0001 \mathrm{mg} / \mathrm{kg}$. NA - not available. WEO-Waste engine oil, MAS - Months after sowing, PS1- soil on which was sown $0.016 \% \mathrm{NaN}_{3}$-treated stem cutting, PS2- $0.064 \% \mathrm{NaN}_{3}$, PS3- $0.250 \% \mathrm{NaN}_{3}$, PX1- soil on which was sown $0.016 \% \mathrm{NH}_{2} \mathrm{OH} . \mathrm{HCl}$-treated stem cuttings, PX2$0.064 \% \mathrm{NH}_{2} \mathrm{OH} . \mathrm{HCl}, \mathrm{PX} 3-0.250 \% \mathrm{NH}_{2} \mathrm{OH} . \mathrm{HCl}$. Means on the same row that are separated by their corresponding LSD values are significantly different from each other $(p<0.05)$. 
Table 3: Polyaromatic hydrocarbon contents of oil- polluted soil after exposure to mutagenic treatments.

\begin{tabular}{|c|c|c|c|c|c|c|c|c|c|c|c|}
\hline \multirow[t]{3}{*}{ PAH components } & \multirow{2}{*}{$\begin{array}{l}\text { Polluted } \\
\text { soil at } \\
\text { Day } 1\end{array}$} & \multirow[b]{2}{*}{$\begin{array}{c}1 \\
\text { month } \\
\text { after } \\
\text { pollutio } \\
n\end{array}$} & \multicolumn{8}{|c|}{3 months after pollution } & \multirow{3}{*}{$\begin{array}{c}\text { LSD } \\
(0.05)\end{array}$} \\
\hline & & & $\begin{array}{c}\text { Unpollut } \\
\text { ed }\end{array}$ & $\begin{array}{l}\text { Polluted } \\
\text { soil }\end{array}$ & PS1 & PS2 & PS3 & PX1 & PX2 & PX3 & \\
\hline & \multicolumn{10}{|c|}{$(\mathrm{mg} / \mathrm{kg})$} & \\
\hline Naphthalene & 29.25 & 25.33 & $\mathrm{Bd}$ & 22.53 & $\mathrm{Bd}$ & $\mathrm{Bd}$ & $\mathrm{Bd}$ & $\mathrm{Bd}$ & $\mathrm{Bd}$ & 3.66 & 3.14 \\
\hline Acenaphthylene & 10.54 & 9.58 & $\mathrm{Bd}$ & 8.63 & $\mathrm{Bd}$ & $\mathrm{Bd}$ & $\mathrm{Bd}$ & $\mathrm{Bd}$ & $\mathrm{Bd}$ & 3.69 & 2.04 \\
\hline 2-bromonaphthalene & 35.21 & 29.03 & $\mathrm{Bd}$ & 29.03 & $\mathrm{Bd}$ & $\mathrm{Bd}$ & $\mathrm{Bd}$ & 15.98 & 16.68 & 4.01 & 3.74 \\
\hline Acenaphthene & 35.46 & 30.51 & $\mathrm{Bd}$ & 28.33 & $\mathrm{Bd}$ & $\mathrm{Bd}$ & $\mathrm{Bd}$ & $\mathrm{Bd}$ & $\mathrm{Bd}$ & 3.92 & 3.43 \\
\hline Fluorene & 45.22 & 40.58 & $\mathrm{Bd}$ & 29.70 & 15.54 & $\mathrm{BDL}$ & $\mathrm{BDL}$ & 19.54 & 16.52 & 3.78 & 3.22 \\
\hline Phenanthrene & 5.62 & 3.68 & 0.28 & 1.29 & 0.08 & 2.68 & 1.77 & 0.96 & 0.29 & 0.03 & 2.31 \\
\hline Anthracene & 29.24 & 28.65 & $\mathrm{Bd}$ & 26.32 & 16.18 & 2.68 & $\mathrm{Bd}$ & 16.95 & 17.22 & 4.36 & 5.23 \\
\hline Fluoranthene & 42.52 & 41.62 & $\mathrm{Bd}$ & 39.99 & $\mathrm{Bd}$ & $\mathrm{Bd}$ & $\mathrm{Bd}$ & $\mathrm{Bd}$ & $\mathrm{Bd}$ & 4.11 & 2.64 \\
\hline Pyrene & 36.20 & 35.98 & $\mathrm{Bd}$ & 32.70 & 15.55 & $\mathrm{Bd}$ & $\mathrm{Bd}$ & 21.93 & 16.66 & 6.26 & 4.93 \\
\hline Benzo(a)anthracene & 53.87 & 55.21 & $\mathrm{Bd}$ & 47.21 & $\mathrm{Bd}$ & $\mathrm{Bd}$ & $\mathrm{Bd}$ & $\mathrm{Bd}$ & $\mathrm{Bd}$ & 9.69 & 2.03 \\
\hline Chrysene & 129.54 & 119.52 & $\mathrm{Bd}$ & 102.04 & $\mathrm{Bd}$ & $\mathrm{Bd}$ & $\mathrm{Bd}$ & $\mathrm{Bd}$ & $\mathrm{Bd}$ & 4.04 & 8.62 \\
\hline $\begin{array}{l}\text { Benzo }(b, j, k) \text { fluorant } \\
\text { hene }\end{array}$ & 59.48 & 52.98 & $\mathrm{Bd}$ & 43.12 & $\mathrm{Bd}$ & 28.28 & 19.34 & 8.56 & 1.88 & 9.75 & 6.03 \\
\hline Benzo(a)pyrene & 209.16 & 185.67 & 23.86 & 183.72 & 19.17 & 18.33 & 20.34 & 54.92 & 30.08 & 31.02 & 11.23 \\
\hline $\begin{array}{l}\text { Indeno(1,2,3- } \\
\text { cd)pyrene }\end{array}$ & 169.54 & 139.54 & 1.24 & 118.42 & 15.74 & 12.54 & 8.93 & 68.20 & 26.85 & 35.05 & 12.69 \\
\hline $\begin{array}{l}\text { Dibenzo(a,h)anthrac } \\
\text { ene }\end{array}$ & 52.20 & 50.67 & 1.33 & 49.81 & 3.07 & 2.65 & 1.89 & 39.25 & 26.61 & 28.38 & 6.52 \\
\hline Benzo( $g, h, i)$ perylene & 72.65 & 68.42 & 9.50 & 66.38 & 22.52 & 24.52 & 23.35 & 32.54 & 28.52 & 42.87 & 8.02 \\
\hline Total & 1015.70 & 916.97 & 36.21 & 829.22 & 107.85 & 91.68 & 91.55 & 278.83 & 181.31 & 194.62 & - \\
\hline Efficiency ( \%) & - & 9.74 & 96.44 & 18.36 & 89.88 & 90.99 & 90.98 & 72.54 & 81.14 & 80.83 & - \\
\hline
\end{tabular}

Bd- Below detectable limit. WEO-Waste engine oil, MAS - Months after sowing, PS1- soil on which was sown 0.016\% NaN 3 -treated stem cutting, PS2- 0.064\% $\mathrm{NaN}_{3}$, PS3- $0.250 \% \mathrm{NaN}_{3}$, PX1- soil on which was sown $0.016 \% \mathrm{NH}_{2} \mathrm{OH}$. HCl-treated stem cuttings, PX2- $0.064 \% \mathrm{NH}{ }_{2} \mathrm{OH} . \mathrm{HCl}, \mathrm{PX} 3-0.250 \% \mathrm{NH} \mathrm{OH}_{2} \mathrm{HCl}$. $\mathrm{Means}$ on the same row that are separated by their corresponding LSD values are significantly different from each other $(p<0.05)$. 
Table 4: Hazard quotient to show toxicity of heavy metal and PAH components of the polluted soil

\begin{tabular}{|c|c|c|c|c|c|c|c|c|c|c|}
\hline \multirow{3}{*}{$\begin{array}{l}\text { Heavy metals } \\
(\mathrm{mg} / \mathrm{kg})\end{array}$} & \multirow{2}{*}{$\begin{array}{l}\text { Polluted } \\
\text { soil at } \\
\text { Day } 1\end{array}$} & \multirow{2}{*}{$\begin{array}{l}1 \text { month } \\
\text { after } \\
\text { pollution }\end{array}$} & \multicolumn{8}{|c|}{3 months after pollution } \\
\hline & & & Unpolluted & $\begin{array}{c}\text { Polluted } \\
\text { soil }\end{array}$ & PS1 & PS2 & PS3 & PX1 & PX2 & PX3 \\
\hline & & & \multicolumn{8}{|c|}{$(\mathrm{mg} / \mathrm{kg})$} \\
\hline \multicolumn{11}{|c|}{ HQ for toxicity of heavy metals to ecosystem } \\
\hline $\mathrm{Cu}$ & 0.41 & 0.35 & 0 & 0.30 & 0.18 & 0.15 & 0.13 & 0.17 & 0.16 & 0.13 \\
\hline $\mathrm{Mn}$ & 0 & 0 & 0 & 0 & 0 & 0 & 0 & 0 & 0 & 0 \\
\hline $\mathrm{Ni}$ & 0 & 0 & 0 & 0 & 0 & 0 & 0 & 0 & 0 & 0 \\
\hline V & 0 & 0 & 0 & 0 & 0 & 0 & 0 & 0 & 0 & 0 \\
\hline $\mathrm{Cr}$ & 20.36 & 18.33 & 0.02 & 16.51 & 0 & 0 & 0 & 10.56 & 9.21 & 7.29 \\
\hline $\mathrm{Pb}$ & 0.37 & 0.33 & 0 & 0.27 & 0.28 & 0.26 & 0.22 & 0.21 & 0.18 & 0.16 \\
\hline \multicolumn{11}{|c|}{ HQ for toxicity of heavy metals to microbial activities and processes } \\
\hline $\mathrm{Cu}$ & 0.16 & 0.14 & 0 & 0.12 & 0.07 & 0.06 & 0.05 & 0.07 & 0.06 & 0.05 \\
\hline $\mathrm{Mn}$ & 0 & 0 & 0 & 0 & 0 & 0 & 0 & 0 & 0 & 0 \\
\hline $\mathrm{Ni}$ & 0 & 0 & 0 & 0 & 0 & 0 & 0 & 0 & 0 & 0 \\
\hline V & 0 & 0 & 0 & 0 & 0 & 0 & 0 & 0 & 0 & 0 \\
\hline $\mathrm{Cr}$ & 2.04 & 1.83 & $2 * 10^{3}$ & 1.66 & 0 & 0 & 0 & 1.05 & 0.92 & 0.72 \\
\hline $\mathrm{Pb}$ & $2 * 10^{-2}$ & $1.8 * 10^{-2}$ & 0 & 0.02 & 0.02 & 0.01 & 0.01 & $1.2 * 10^{-2}$ & $1.0 * 10^{-2}$ & $9.0 * 10^{-3}$ \\
\hline \multicolumn{11}{|c|}{ HQ for toxicity of polyaromatic hydrocarbon components to ecosystem } \\
\hline Naphthalene & 292.5 & 253.30 & 0 & 225.30 & 0 & 0 & 0 & 0 & 0 & 36.60 \\
\hline Acenaphthene & 1.77 & 1.53 & 0 & 283.30 & 0 & 0 & 0 & 0 & 0 & 0.19 \\
\hline Fluorene & 1.51 & 1.35 & 0 & 297.00 & 0.52 & 0 & 0 & 0.65 & 0.55 & 0.13 \\
\hline Phenanthrene & 5.62 & 36.80 & 2.80 & 12.90 & 0.80 & 26.80 & 177.00 & 9.60 & 2.90 & 0.30 \\
\hline Anthracene & 292.40 & 286.50 & 0 & 263.20 & 161.80 & 26.80 & 0 & 169.50 & 172.2 & 43.60 \\
\hline Fluoranthene & 425.20 & 416.20 & 0 & 399.90 & 0 & 0 & 0 & 0 & 0 & 41.10 \\
\hline Pyrene & 538.70 & 359.80 & 0 & 327.00 & 155.50 & 0 & 0 & 219.30 & 166.60 & 62.20 \\
\hline Benzo(a)pyrene & 209.16 & 1856.70 & 233.80 & 1837.20 & 191.70 & 183.30 & 203.40 & 549.20 & 300.80 & 310.20 \\
\hline
\end{tabular}

HQ > 1 indicates toxicity. Toxicity benchmarks for whch HQ were obtained are provided by Efroymson et al. (1997). WEO-Waste engine oil, MAS - Months

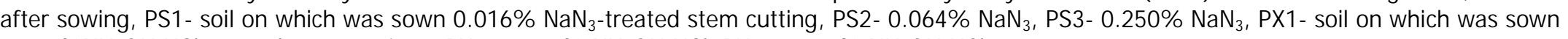
$0.016 \% \mathrm{NH}_{2} \mathrm{OH} . \mathrm{HCl}$-treated stem cuttings, PX2- $0.064 \% \mathrm{NH}_{2} \mathrm{OH} . \mathrm{HCl}, \mathrm{PX} 3-0.250 \% \mathrm{NH}_{2} \mathrm{OH} . \mathrm{HCl}$. 
Table 5: Toxicity equivalency concentration of the polyaromatic hydrocarbon contents of polluted soil after exposure to mutagenic treatments.

\begin{tabular}{|c|c|c|c|c|c|c|c|c|c|c|}
\hline \multirow[t]{3}{*}{ PAH components } & \multirow{2}{*}{$\begin{array}{c}\text { Polluted } \\
\text { soil at Day } \\
1\end{array}$} & \multirow{2}{*}{$\begin{array}{l}1 \text { month } \\
\text { after } \\
\text { pollution }\end{array}$} & \multicolumn{8}{|c|}{3 months after pollution } \\
\hline & & & Unpolluted & $\begin{array}{c}\text { Polluted } \\
\text { soil }\end{array}$ & PS1 & PS2 & PS3 & PX1 & PX2 & PX3 \\
\hline & \multicolumn{10}{|c|}{$(\mathrm{mg} / \mathrm{kg})$} \\
\hline Benzo(a)anthracene & 5.38 & 5.52 & 0 & 47.21 & 0 & 0 & 0 & 0 & 0 & 9.69 \\
\hline Chrysene & 1.29 & 1.19 & 0 & 1.02 & 0 & 0 & 0 & 0 & 0 & 0.04 \\
\hline Benzo(b,j,k)fluoranthene & 209.16 & 185.67 & 23.86 & 183.72 & 19.17 & 18.33 & 20.34 & 54.92 & 30.08 & 31.02 \\
\hline Benzo(a)pyrene & 16.95 & 13.95 & 0.12 & 11.84 & 1.57 & 1.25 & 0.89 & 6.82 & 2.66 & 2.84 \\
\hline Indeno(1,2,3-cd)pyrene & 238.72 & 206.33 & 23.98 & 243.79 & 20.74 & 19.58 & 21.23 & 61.74 & 32.74 & 43.59 \\
\hline TOTAL TEQ & 471.5 & 412.66 & 47.96 & 487.58 & 41.48 & 39.16 & 42.46 & 123.48 & 65.48 & 87.18 \\
\hline
\end{tabular}

WEO-Waste engine oil, MAS - Months after sowing, PS1- soil on which was sown $0.016 \% \mathrm{NaN}_{3}$-treated stem cutting, PS2- $0.064 \%$ NaN 3 , PS3- $0.250 \%$ NaN ${ }_{3}$, PX1- soil on which was sown $0.016 \% \mathrm{NH}_{2} \mathrm{OH}$. HCl-treated stem cuttings, PX2- $0.064 \% \mathrm{NH}_{2} \mathrm{OH}$. $\mathrm{HCl}, \mathrm{PX} 3-0.250 \% \mathrm{NH}_{2} \mathrm{OH} . \mathrm{HCl}$. 
Achromobacter sp, Micrococcus varians and $P$.aeruginosa were relatively dorminant bacteria species in that order (Table 6). Total heterotrophic bacteria count was $7.6 \times 10^{5} \mathrm{cfu} / \mathrm{g}$ in the unpolluted soil after sowing Chromoleana. In PS-soils, heterotrophic bacteria count ranged from $4.2-6.5 \times 10^{5}$ $\mathrm{cfu} / \mathrm{g}$, and $3.8-5.8 \times 10^{5} \mathrm{cfu} / \mathrm{g}$ in PX-soils. Comparatively increase concentration of the mutagenic agent decreased heterotrophic bacteria composition. However, increase concentration of mutagenic solution increased percentage composition of hydrocarbon degrading fungi from $244.7 \%$ in PS1 to $71.4 \%$ in $\mathrm{PS3}$, and from $46.2 \%$ in PX1 to $61.9 \%$ in $P X 3$. Example of hydrocarbon degrading fungi found in this study were Aspergillus niger (most prevalent), A.Flavus, Penicillium sp, Fusarium Solani.

Number of sprouted branches per plant stand at 2 months after sowing (MAS) for all treatment was highest at PX1 with 5 branches and lowest at PS3 with 2 branches (Table 6). Total number of leaves was 68 leaves per plant at PX1 and 27 leaves at PS3. Whereas necrotic leaves per plant at 2 MAS were 6 leaves at PS1 and 4 leaves at PX3.

Table 6: Microbial composition of soil at 3 months after pollution

\begin{tabular}{|c|c|c|c|c|c|c|c|c|}
\hline \multirow[t]{2}{*}{ PAH components } & \multicolumn{2}{|c|}{ Control } & \multirow[b]{2}{*}{ PS1 } & \multirow[b]{2}{*}{ PS2 } & \multirow[b]{2}{*}{ PS3 } & \multirow[b]{2}{*}{ PX1 } & \multirow[b]{2}{*}{ PX2 } & \multirow[b]{2}{*}{ PX3 } \\
\hline & $\begin{array}{l}\text { Unpollut } \\
\text { ed }\end{array}$ & $\begin{array}{l}\text { Pollute } \\
\text { d soil }\end{array}$ & & & & & & \\
\hline \multicolumn{9}{|c|}{ Bacterial species } \\
\hline Achromobacter sp & + & + & + & + & - & + & + & - \\
\hline * Micrococcus varians & + & + & + & + & + & + & + & + \\
\hline M. roseus & - & + & + & + & - & + & - & - \\
\hline *Bacillus pumilis & + & - & + & + & - & + & + & - \\
\hline B. subtilis & + & + & + & - & - & + & - & - \\
\hline Pseudomonas sp & - & . & + & - & + & + & + & - \\
\hline *P. aeruginosa & + & + & + & + & + & + & - & - \\
\hline $\begin{array}{l}\text { Heterotrophic bacteria (x } 10^{5} \\
\mathrm{cfu} / \mathrm{q} \text { ) }\end{array}$ & 7.6 & 5.6 & 6.5 & 5.6 & 4.2 & 5.8 & 4.5 & 3.8 \\
\hline $\begin{array}{l}\text { Hyd. Deg. bacteria (x } 10^{5} \\
\text { cfu/g) }\end{array}$ & 4.9 & 3.9 & 3.8 & 3.2 & 2.6 & 3.4 & 2.5 & 1.7 \\
\hline$\%$ Hyd & 64.5 & 69.6 & 58.5 & 57.1 & 61.9 & 58.6 & 55.6 & 44.7 \\
\hline \multicolumn{9}{|c|}{ Fungal species } \\
\hline *Aspergillus niger & + & + & + & - & + & + & - & - \\
\hline$*$ A. Flavus & + & + & + & - & - & + & - & - \\
\hline *Penicillium sp & + & - & + & + & - & + & + & - \\
\hline *Fusarium solani & - & - & - & + & + & - & - & + \\
\hline Mucor sp & + & + & - & - & - & + & - & + \\
\hline Geotrichum sp & + & + & + & - & - & + & - & - \\
\hline Trichoderma sp & + & + & - & - & - & - & - & - \\
\hline $\begin{array}{l}\text { Heterotrophic Fungi ( } \times 10^{5} \\
\mathrm{cfu} / \mathrm{g} \text { ) }\end{array}$ & 4.9 & 3.8 & 3.8 & 3.2 & 2.8 & 3.9 & 3.2 & 2.1 \\
\hline Hyd. deg. Fungi (x $\left.10^{5} \mathrm{cfu} / \mathrm{g}\right)$ & 2.7 & 1.8 & 1.7 & 1.5 & 2.0 & 1.8 & 1.6 & 1.3 \\
\hline$\%$ Hyd & 55.1 & 47.4 & 44.7 & 46.9 & 71.4 & 46.2 & 50 & 61.9 \\
\hline
\end{tabular}

+present, -absent, *hydrocarbon degraders. WEO-Waste engine oil, MAS - Months after sowing, PS1soil on which was sown $0.016 \% \mathrm{NaN}_{3}$-treated stem cutting, PS2- $0.064 \% \mathrm{NaN}_{3}, \mathrm{PS} 3-0.250 \% \mathrm{NaN}_{3}$, PX1- soil on which was sown $0.016 \% \mathrm{NH}_{2} \mathrm{OH}$. HCl-treated stem cuttings, PX2- $0.064 \% \mathrm{NH}_{2} \mathrm{OH} . \mathrm{HCl}, \mathrm{PX} 3-$ $0.250 \% \mathrm{NH}_{2} \mathrm{OH} . \mathrm{HCl}$. 
Table 6: Selected plant parameters at 2 months after sowing

\begin{tabular}{|c|c|c|c|c|c|c|c|c|c|}
\hline \multirow[t]{2}{*}{ Plant parameter } & \multicolumn{2}{|c|}{ Control } & \multicolumn{3}{|c|}{$\mathrm{NaN}_{3}$ solution } & \multicolumn{3}{|c|}{$\mathrm{NH}_{2} \mathrm{OH} . \mathrm{HCl}$ solution } & \multirow{2}{*}{$\begin{array}{c}\text { LSD } \\
(0.05 \\
\quad)\end{array}$} \\
\hline & Unpolluted & $\begin{array}{l}\text { Pollute } \\
\text { d soil }\end{array}$ & PS1 & PS2 & PS3 & PX1 & PX2 & $\mathrm{PX3}$ & \\
\hline No. Of sprouted branches & 3.2 & 4.1 & 3.1 & 2.8 & 2.2 & 5.3 & 3.2 & 4.3 & 0.7 \\
\hline Leaf area $\left(\mathrm{cm}^{2}\right)$ & 9.4 & 3.2 & 4.3 & 5.3 & 7.0 & 6.6 & 4.9 & 9.8 & 2.1 \\
\hline Length of sprouted branch $(\mathrm{cm})$ & 32.2 & 15.5 & $\begin{array}{c}13 . \\
5\end{array}$ & 12.3 & 16.0 & 17.6 & 16.0 & 16.2 & 5.1 \\
\hline *Total No. Of leaves & 48.0 & 37.0 & $\begin{array}{c}28 . \\
1\end{array}$ & 19.2 & 27.0 & 68.0 & 42.0 & 45.0 & 4.8 \\
\hline No. Of necrotic leaves per plant & 0 & 0 & 6.3 & 0 & 0 & 0. & 0 & 4.2 & 1.6 \\
\hline Total No. of senesced leaves & 7.0 & 13.0 & 8.0 & 12 & 11.0 & 8.0 & 11.0 & 15.0 & 2.2 \\
\hline $\begin{array}{l}\text { No. Of reddish brown leaves per } \\
\text { plant }\end{array}$ & 0 & 4.0 & 4.0 & 0 & 6.0 & 10.0 & 4.0 & 8.0 & 2.8 \\
\hline
\end{tabular}

* This includes total number of scenesced leaves. DAS- Days after sowing, WEO-Waste engine oil, MAS - Months after sowing, PS1- soil on which was sown $0.016 \% \mathrm{NaN}_{3}$-treated stem cutting, PS2- $0.064 \% \mathrm{NaN}_{3}, \mathrm{PS} 3-0.250 \% \mathrm{NaN}_{3}$, PX1- soil on which was sown $0.016 \% \mathrm{NH}_{2} \mathrm{OH}$. $\mathrm{HCl}$-treated stem cuttings, PX2- $0.064 \% \mathrm{NH}_{2} \mathrm{OH} . \mathrm{HCl}, \mathrm{PX} 3-0.250 \%$ $\mathrm{NH}_{2} \mathrm{OH} . \mathrm{HCl}$. Means on the same row that are separated by their corresponding LSD values are significantly different from each other $(p<0.05)$.

\section{Discussion}

Reseults of this study showed that pollution of soil by WEO brought about increased concentration of heavy metals in the soil. However, introduction of plants resulted significantly in the reducution of heavy metal concentrations of the soil.Results also showed that with the increase in concentration of mutagenic solutions required for presoaking, total heavy metal concentration in soil decreased. Total heavy metal was least in PS3 (16.17 mg/kg), compared to $21.04 \mathrm{mg} / \mathrm{kg}$ as total in PS1, $28.43 \mathrm{mg} / \mathrm{kg}$ in PX1 and 20.43 $\mathrm{mg} / \mathrm{kg}$ in $\mathrm{PX}$.

Metals are amongst mineral nutrients needed by plant, but at higher concentration all metal are toxic due to the fact that they form free radical which causes oxidative stress. Also toxicity of metals is attributed to the ability of certain metals to be metabolism or take up by plant roots in place of necessary metals in enzymes and pigment reactions causing functtional disruption.(Henry, 2000). However, certain plants thrive in polluted environments as have been established by earlier studies (Wong and Chu, 1985; Anoliefo and Edegbai, 2001; Dede et al., 2003; Olivares, 2003; Ogboghodo et al.,2001; Vwioko and Fashemi, 2005), in Ricinus communis. Anoliefo et al $(2006,2008)$ also identified Chromolaena in heavy metal contaminated soils. These plants are phytoremediative and phytoremediation provides superior benefit for the remediation of heavy metal. In the present study, Chromolaena plants that had been pretreated in concentrations of sodium azide caused better heavy metal remediation than those preteated with hydroxylamine hydrochloride. For example, $\mathrm{Cr}$ was below detectable limit when oil-polluted soil was sown with sodium azide-preteated stem cuttings.

Chromolaena odorata has been reported from previous research to have ability to tolerate oil (Anoliefo and Vwiko, 2001), has high ash content and can therefore bioaccumulate and translocate hydrocarbon compounds and heavy metals (Belford et al.,2009). From this research it can be observed that Chromolaena odorata remediated the waste engine oil polluted soil for both poly aromatic hydrocarbon and heavy metals. Poly aromatic hydrocarbons naphthalene, acenaphthylene, acenaphthene, fluoranthene, benzo(a)anthracene, chrysene were all remediated below detectable limits in treatments with $\mathrm{NaN}_{3}(0.016 \%, 0.064 \%$, $0.25 \%), \quad \mathrm{NH}_{2} \mathrm{OH} . \mathrm{HCl} \quad(0.016 \%, \quad 0.064 \%)$ respectively. There was also significant reduction in the concentration of benzo[b]fluoranthene, benzo(ghi)perylene, benzo[k]fluoranthene, dibenzo[a, $h]$ anthracene at the various treatments. These PAH components were hitherto present in the soil before pollution and increased with pollution by waste engine oil. The activities of soil microbes have been noted to help PAH remediation greatly particularly in collaboration with plant roots (Anoliefo and Ikhajiagbe, 2011). 
Microbial degradation of light weight aromatic hydrocarbon, which are easily evaporated are accessable to microbes in soluble or dissolved forms (Jordan and Payne, 1980; Kappeler and Wuhrmann, 1978). Hydrocarbon degradation is not limited to few genera of microbes, but the ability to degrade has been observed in a varied group of fungi and bacteria.In the present study, Achromobacter sp., Micrococcus varians and $\mathrm{P}$. Aeruginosa were the predominant bacterials species, whereas A. Niger and Penicillium sp. were predominant fungi. Occurence of $P$. Aeruginosa was $100 \%$ in all PS-treatments, compared to 1-in-3 PX treatments. Incidently, it was identified as an oil degrader in this study. That may perhaps explain the enhanced heavy metal and PAH reediations in the PS treatments, compared to the PX. Similartly, A. niger was present in 2-in-3 PS-treatements, as against 1-in-3 PX treatments.

There was however greater remediation in the mutagenic pretreated stems of Chromolaena odorata in all treatments and total remediation of most poly aromatic hydrocarbon with some treatment of the mutagenic agents, as seen in naphthalene, acenaphthylene, acenaphthene, fluoranthene, benzo(a)anthracene, chrysene, which were all totally remediated in treatments with $\mathrm{NaN}_{3}$ $(0.016 \%, \quad 0.064 \%, \quad 0.25 \%), \quad \mathrm{NH}_{2} \mathrm{OH} . \mathrm{HCl}$ $(0.016 \%, 0.064 \%)$. Mutagenic solutions may have enhanced the plant's capacity for remediation. Mensah and Akomeah (1992); Mensah et al. (2006) and Mshembula et al (2012) reported enhanced plant growth and development as a result of pretreatment with sodium azide and hydroxylamine hydrochloride. I khajiagbe et al (2013) reported that enhanced plant growth of sodium azide treated rice exposed to waste engine oil pollution. Though the introduction of mutagens increased the growth response of stem cuttings and improved the bioaccumulation rate of Chromolaena odorata, it also increased the metabolic rate of the plant, bringing about early senescence and also death of the plant that were pretreated with the mutagens.

The leaves of Chromolaena odorata plant on the polluted soil all showed reddish-brown discoloration of leaves starting from leaf tip and progressing inward. This symptom is mainly consistent with phosphorus deficiencies which exhibits red or purple pigmentation as a result of anthocyanin pigment. Phosphorus is a component of certain enzymes and protein, adenosine triphosphate. Reddish brown coloration is also associated with $\mathrm{N}, \mathrm{K}, \mathrm{Mg}$ deficiencies in plant. Oil polluted soil are prone to nutrient deficiency, which may indirectly result from reduced water uptake from clogging of soil pore by the presence of waste engine oil in the soil. According to (Udo and Fayemi, 1975) soil contaminated by oil may be inappropriate for farming as a result of nutrient avaliability reduction or due to increase in toxic concentration of heavy metals such as manganese. The presence of these heavy metals in oil polluted soil causes inhibition of growth and disruption of metabolic processes in plants.

\section{Conclusion}

Phytoremediation is a useful and effecitive method of cleaning contaminants from the environment, although it is slow and usually requires a time frame to be very effecitive. This prolong or waiting period for efficiency makes it a tedious technique useful by environmentalise. The present study shows that the intoduction of mutagenic agent such as sodium azide and hydroxylamide to planting materials help not only to aid rapid growth of Chromolaena odorata (Siam weed), but also help the with the bioaccumulative potential of Chromolaena odorata (Siam weed), as seen in the treatment of various concentrations of sodium azide and hydroxylamide compared with the control without any treatment. There was great reduction in PAH and heavy metal component of the soil.

\section{Acknowledgement}

The reserachers are grateful to Prof. J.K. Mensah of the Department of Botany, Ambrose Alli University, Ekpoma, for his support.

\section{References}

Akoachere, T. K. Jane-Francis., Akenji, N. Theresa., Yongabi, N. Felicitas., Nkwelang, G., and Ndip, R.N. (2008). Lubricating oildegrading bacteria in soils from filling stations and auto- mechanic workshops in Buea, Cameroon: occurrence and characteristics of isolates. African Journal of Biotechnology, 7 :1700-1706.

Anoliefo, G.O. and Edegbai, B.O. (2001). Effect of spent engine oil as a soil contaminant on the growth of two egg plant species, Solanum melongen $\mathrm{L}$ and $\mathrm{S}$. incanum. Journal of Agriculture, Forestry and Fisheries, 1:21-25.

Anoliefo, G.O and Ikhajiagbe, B. (2011). Plantmicrobial interaction in the degradation of 
crude oil in soil: Synergism in bioremediation. Nigerian Journal of Life Sciences 1(1): 40-52.

Anoliefo, G.O., and Vwioko, D.E. (2001). Tolerance of Chromolaena odorata (L) K \& R. grown in soil-contaminated with spent lubricating oil. Journal of Tropical Biosciences, 1: 20-24.

Anoliefo, G.O., Ikhajiagbe, B., Okonokhua, B.O. and Diafe, F.V. (2006). Ecotaxonomic distribution of plant species around auto mechanic workshops in Asaba and Benin City: Identification of oil tolerant species. African Journal of Biotechnology, 5(19):1757-1762.

Anoliefo, G.O; Ikhajiagbe, B; Okonokhua, B.O; Edegbai, B.E; and Obasuyi, O.C (2008). Metal tolerant species distribution and richness in and aroud metal based industries: possible candidates for phytoremediation. Africa Journal of Environmental Science and Technology, 2 (11): 360-370.

Belford, J.D., Aziz, F., Baidoo, P.K. (2009): Phytoremediation of heavy metal contaminated soil using Chromolaena odorata and Lantana camara. MSc. Department of Theoretical and Applied Biology, Kwame Nkrumah University of Science and Technology, Ghana.

Bossert, I. and Bartha, R. (1984). The fate of petroleum in the ecosystem. In: Atlas, R. M. (ed). Petroleum Microbiology. Macmillan, new York pp. 435-475.

Cheesebrough, M. (1998). District laboratory practice in tropical countries, part II (Microbiology). Cambridgeshire Tropical Health Technology, Cambridge, UK.

Cowan, S. T. and Steele, K. J., (1974). Manual for Identification of Medical Bacteria. 2nd. Ed., Cambridge University Press, Cambridge, UK. $216 p$.

Dede, E.B., Igboh, N.M., and Ayalogu, O.E. (2003). Ecotoxicological effect of crude oil (Bonny Light), kerosene and gasoline on Celosia argentia plant. Journal of Nigerian Environmental Society: 1: 131-136.

Efroymson, R.A., Will, M.E., Suter II, G.W. and Wooten, A.C. (1997). Toxicological Benchmarks for Screening Contaminants of Potential Concern for Effects on Terrestrial Plants: 1997 Revision. ES/ER/TM-85/R3. U.S. Department of Energy, Office of Environmental Management, 123p.
Henry, J. R. (2000). An Overview of Phytoremediation of Lead and Mercury. NNEMS Report. Washington, D.C. pp, 3-9.

Ibekwe, V. I., Ubochi, K. C., and Ezeji, E. U. (2006). Effect of organic nutrient on microbial utilization of hydrocarbons on crude oilcontaminated soil. African Journal of Biotechnology 5: 983-986.

Ikhajiagbe, B. (2010). Synergism in Bioremediation: Phytoassessment of Waste Engine Oil Polluted Soils after Amendment and Bioaugmentation. LAP Lambert Academic Publishing, Köln, Germany. 276p

Ikhajiagbe, B., Odigie, E. Ujomonigho., Okoh, B. Efenaide., and Agho, E. Esther. (2013). Effects of sodium azide on the survival, growth and yield performance of rice (Oryza sativa, FARO-57 variety) in a hydrocarbon-polluted soil. The International J ournal of Biotechnology 2(1):28-41.

Jordan, B. and Payne, J. R. (1980). Fate and Weathering of Petroleum Spills in the Marine Environment. Ann Arbor Science Publications Inc., Ann Arbor, Michigan. 344p.

Kappeler, Th. and Wuhrmann, K. (1978). Microbial degradation of the water-soluble fraction of gas oil-11. Bioassays with the Pure Strains of Water Research, 12:335-342.

Khan, A. G., Kuek, C., Chaudhry, T. M., Khoo, C. S., and Hayes, W. J. (2000). Role of plants, mycorrhizae and phytochelators in heavy metal contaminated land remediation. Chemosphere 41:197-207.

Kharkwal, M.C. and Shu, Q.Y. (2009). The Role of Induced Mutations in World Food Security, pp. 33-38. In: Shu, Q.Y. (ed.) Induced Plant Mutations in the Genomics Era, Food and Agriculture Organization of the United Nations, Rome, I taly.

Meagher, R. B. (2000). Phytoremediation of toxic elemental organic pollutants. Current Opinon Plant Biology. 3:162.

Mensah, J.K. and Akomeah, P.A. (1992) Mutagenic effects of hydroxylamine and streptomycin on the growth and seed yield of cowpea (Vigna unguiculata (L.) Walp). Legume Research 15(1): 39-44. 
Mensah, J.K., Obadoni, B.O., Akomeah, P.A., Ikhajiagbe, B. and Ajibolu, Janet (2006). Effects of sodium azide and colchicines treatments on morphological and yield traits of sesame seed (Sesame indicum L.). African Journal of Biotechnology 6(5): 534-538.

Mshembula, B.P., Mensah, J.K and Ikhajiagbe, B (2012).Comparative assessment of the mutagenic effects of sodium azide on some selected growth and yield parameters of five accessions of cowpea - Tvu-3615, Tvu-2521, Tvu-3541, Tvu-3485 and Tvu-3574. Archives of Applied Science Research 4 (4):1682-1691.

Odu, C.T.I. (1972). Microbiology of soils contaminated with petroleum hydrocarbon I. Extent of contamination and some soil and microbial properties after contamination. Journal Institutione Pasteur, 58: 201-208.

Ogboghodo, I.A., Iruafa, E.K., Osenwota, I.O. and Chokor, J.U. (2001). An assessment of the effect of crude oil pollution on soil properties: germination and growth of maize. Proceedings of the 27th Annual Conference of the Soil Science Society of Nigeria Nov 5th - 9th 2001, University of Calabar, Nigeria.

Raven, R., Berg, L. R., and Johnson, G. B. (1993). Environment. Saunders College Publishing, Philadelphia. USA, p. 569.
Udo, E.J. and. Fayemi, A.A.A. (1975). The effect of oil pollution on germination, growth and nutrient uptake of corn. Journal of Environmental Quality, 4:537-540.

Vwioko, D.E. and Fashemi, D.S. (2005). Growth response of Ricinus communis $L$ (Castor Oil) in spent lubricating oil-polluted soil. Journal of Applied Sciences and Environmental Management, 9: 73-79.

Wang, Q., Cui, Y., and Dong, Y. (2002). Phytoremediation of polluted waters potential and prospects of wetland plants. Acta Biotechnology, 22(1-2): 199-208.

Whisman, M.L., Geotzinger, J.W., and Cotton, F.O. (1974). Waste lubricating oil research. In: An Investigation of several Re-refining Methods. Bureau of Mines. Bartlesville. Energy Research Center, 352p.

Wong, M. H. and Chu, L.M. (1985). Yield and metal uptake of Cynodon dactylon (Bermuda grass) grown in refuse-compost-amended soil. Agriculture, Ecosystems and Environment, 14 (1-2): 41-52. 